\title{
Is inter-basin groundwater exchange required in rainfall-runoff models: The Australian context
}

\author{
J. D. Hughes ${ }^{\text {a }}$, N. J. Potter ${ }^{\mathrm{a}}$ and L. Zhang ${ }^{\mathrm{a}}$ \\ ${ }^{a}$ CSIRO Land and Water, Canberra, Australia \\ Email: justin.hughes@csiro.au
}

\begin{abstract}
Inter-catchment groundwater exchange is a process included in some rainfall runoff models, most notably GR4J, to account for transfer of groundwater in and out of catchments. This process has been validated in European conditions, but not in Australia. It is unknown if inter-catchment groundwater exchange can be justified in Australian environments. The rainfall-runoff models GR4J and GR7J are outlined. Simulated intercatchment groundwater fluxes for 137 calibrated catchments from each model show that this term comprises a significant part of the simulation water balance. In a random sub-sample of catchments $(n=30)$, the intercatchment groundwater exchange parameter $\left(x_{2}\right)$ is set to zero (effectively shutting off the process). GR4J and GR7J were re-calibrated, and evaluated with this modification during identified extended drought periods. Results suggest that the $x_{2}$ parameter can be set to zero for the GR7J model without any deterioration in predictive performance. GR4J goodness of fit is degraded in both calibration and evaluation by setting $x_{2}$ to zero. Catchment water balance is evaluated for 137 catchments within eastern Australia using observations of precipitation, potential evaporation and stream flow. Analysis of these data could not detect imbalances that suggest significant inter-catchment groundwater exchange. It is proposed that inter-catchment groundwater exchange process in a calibrated rainfall-runoff cannot be justified in the study conditions without additional contrary evidence.
\end{abstract}

Keywords: Drought, rainfall-runoff model, non-stationarity, groundwater-surface water connection, objective functions 
J. D. Hughes et al., Inter-basin groundwater exchange...

\section{INTRODUCTION}

Conceptual rainfall-runoff models are, at their simplest, a way of transforming a climate input into a stream flow output. Within this broad definition the number and nature of rainfall-runoff models varies widely, since each was developed with different conceptual understanding and different intended model uses. Some models may be treated as a "black-box", where no importance is placed on internal processes. Since rainfallrunoff models are calibrated, they may be expected to perform adequately within the bounds of calibration conditions. However, investigations the effect of changing climatic inputs on runoff often leads to forecasts outside of calibration conditions (e.g. Vaze et al., 2010), and poor prediction may occur.

Model structural changes to represent long-term storage changes and ephemeral runoff production in Australian catchments were introduced in the GR7J model by Hughes et al. (2015), with subsequent improvement in predictions during drought conditions. The $x_{2}$ parameter in the GR4J and GR7J controls the loss or gain of water from the routing store. Conceptually, this process is intended to represent exchange of groundwater with surrounding catchments. These processes have been tested widely in over 1000 catchments in France by Le Moine et al. (2007). The authors concluded that without the inclusion of such processes, some simulations would require adjustments to climate and streamflow observations that may yield unrealistic simulated fluxes. However, the possibility exists that in Australian conditions (geology, drier, low relief), that groundwater exchange process may simply operate as a "fitting process". If so, other simulated internal processes ( particularly evapo-transpiration) may be unrealistic, thereby increasing the chances of over-fitting and poor predictive performance, viz. streamflow simulation (during calibration) has acceptable goodness of fit but for the wrong reasons.

Here we investigate the possibility of removing the groundwater exchange process from both GR4J and GR7J and examine what effects this has on predictive performance and model states.

\section{METHODS}

\subsection{Site information}

The study area and catchments used for this study are the same as those used by Saft et al. (2015). The 137 catchments selected are largely free of contriving factors such as large reservoirs, substantial irrigation diversion or land use change. The catchments are located in close proximity to the great dividing range along the south-eastern coastline of the Australian mainland. These areas produce much of the runoff in south-eastern Australia including the Murray-Darling basin (Figure 1). Median annual runoff for the study catchments is $155 \mathrm{~mm}$, while median annual precipitation is $870 \mathrm{~mm}$ and median annual potential evaporation is $1165 \mathrm{~mm}$.

\subsection{Drought classification}

Long-term drought periods were classified using annual rainfall. Annual rainfall anomaly data were calculated relative to the annual mean, the anomaly series was divided by the mean annual rainfall and smoothed with a 3 year moving window. To ensure that the dry periods are sufficiently long and severe, in the subsequent analysis we only used dry periods that were 7 or more years long, and, with a mean dry period anomaly of $<-5 \%$. The millennium drought (late 1990's - 2009) was the main and usually the only long-term drought period identified for most catchments. For further information see Saft et al. (2015).

\subsection{Model framework}

The GR4J and GR7J models was used as a test-bed for model structural changes. The GR4J model (Perrin et al., 2003), is widely used and is relatively simple in nature. It consists of two main stores: the production store $(S)$, and the routing store $(R)$. Net rainfall $\left(P_{n}\right)$ and net evaporation $\left(E_{n}\right)$ were calculated as follows;

$$
P_{n}=\max \left(0, P_{i}-P E_{i}\right)
$$

and

$$
E_{n}=\max \left(0, P E_{i}-P_{i}\right)
$$

where $P_{i}$ was the precipitation on day $i$ and $P E_{i}$ was the potential evaporation on day $i$. 
J. D. Hughes et al., Inter-basin groundwater exchange...

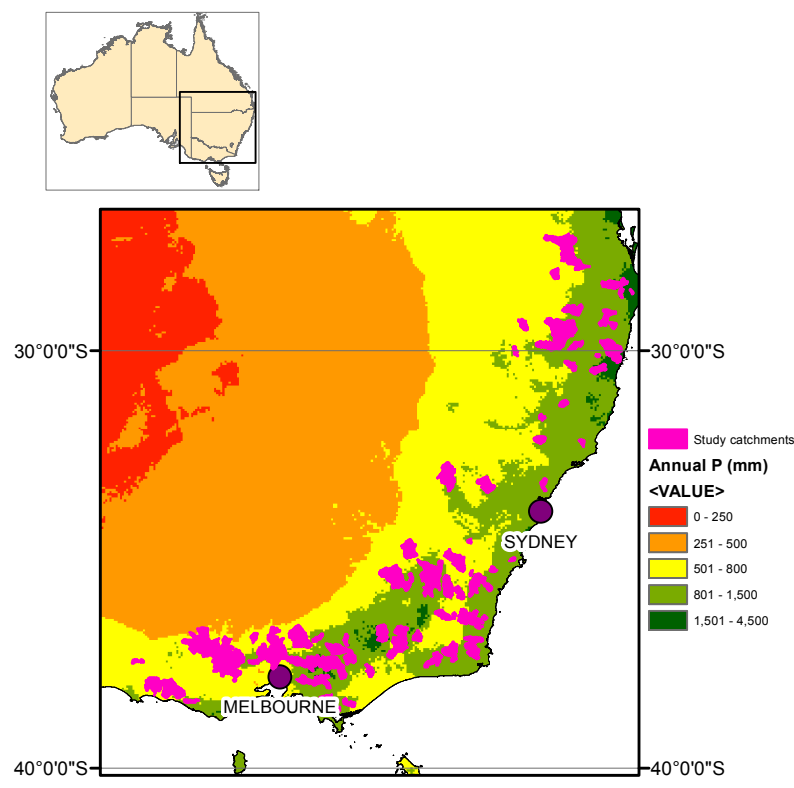

Figure 1. Location of study catchments

$P_{s}$ is the amount on net rainfall that is added to the production store. This amount depends upon both net rainfall $P_{n}$, and the current state of the production store $S$;

$$
P_{s, G R 4 J}=\frac{x_{1}\left(1-\left(\frac{S}{x_{1}}\right)^{2}\right) \tanh \left(\frac{P_{n}}{x_{1}}\right)}{1+\frac{S}{x_{1}} \tanh \left(\frac{P_{n}}{x_{1}}\right)}
$$

where $S$ is the current level of storage ( $\mathrm{mm}), P_{n}$ is the net precipitation (mm), and $x_{1}$ is the maximum storage of $S$, and is a calibrated parameter. Net rainfall in excess of $P_{s, G R 4 J}$ is routed to runoff;

$$
P_{r}=P_{n}-P_{s}
$$

where $P_{r}$ is the amount of net rainfall that becomes available for routing to runoff.

In a similar way, evaporative loss from the production store $\left(E_{s}\right)$ is calculated relative to the level of the production store $(S)$;

$$
E_{s, G R 4 J}=\frac{S\left(2-\frac{S}{x_{1}}\right) \tanh \left(\frac{E_{n}}{x_{1}}\right)}{1+\frac{S}{x_{1}} \tanh \left(\frac{E_{n}}{x_{1}}\right)}
$$

where $E_{n}$ is net evaporation (Equation 2). The GR7J model code is an adaption of GR4J with the aim of allowing more flexibility in the way the processes encapsulated in Equations 3 and 5 relate to the state of the production store $(S)$.

To vary the sensitivity of $P_{s}$ to $S$, the exponent in the numerator of Equation 3, was re-coded in GR7J as a calibrated parameter of value between 1 and 3;

$$
P_{s, G R 7 J}=\frac{x_{1}\left(1-\left(\frac{S}{x_{1}}\right)^{x_{5}}\right) \tanh \left(\frac{P_{n}}{x_{1}}\right)}{1+\frac{S}{x_{1}} \tanh \left(\frac{P_{n}}{x_{1}}\right)}
$$

where $x_{5}$ is the new calibrated parameter.

The updated $E_{s}$ calculation for GR7J required two additional calibrated parameters $\left(x_{6}\right.$ - magnitude factor and $x_{7}$ - shape factor), and a user defined value (expand) which determines the size of the available depletion 
J. D. Hughes et al., Inter-basin groundwater exchange...

relative to the size of the production store available for runoff production $\left(x_{1}\right)$;

$$
E_{s, G R 7 J}=E_{n} * x_{6} *\left(\frac{\left(S_{\max }-x_{1}+S\right)}{S_{\max }}\right)^{x_{7}}
$$

where $S_{\max }=$ expand $* x_{1}$ and expand has a value greater than or equal to 1 . Effectively, a value of expand $>1$ allows depletion of the production store to continue via evaporative processes when all incoming precipitation $P_{n}$ is captured by the production store and none is routed to streamflow. In this study an expand value of 2.0 was used.

\subsection{Switching off the groundwater exchange process}

The groundwater exchange process is controlled by the following relationship:

$$
F_{i}=x_{2} *\left(\frac{R_{i}}{x_{3}}\right)^{3.5}
$$

where $F$ is flux of water moving in or out of the routing store, $x_{3}$ is a calibrated parameter controlling the size of the routing store, and $R_{i}$ is the storage level of the routing store on day $i$. On a random sub-sample of 30 catchments used to assess GR7J and GR4J from the study of Hughes et al. (2015), the $x_{2}$ parameter was set to zero and calibrated with the $N S E_{S e g}$ objective function and evaluated in drought periods as specified using drought classification scheme of Saft et al. (2015). This was compared to the same catchments where the $x_{2}$ parameter was calibrated. The upper calibration bound for parameter $x_{5}$ was increased from 3 to 5 since it was noticed that optimisation with $x_{2}$ set to zero resulted in catchments having calibrated values of the upper bound when it was set at 3 .

\subsection{Model calibration}

An objective function was used where absolute bias was calculated for 365 day segments of the simulated and observed time series:

$$
N S E_{S e g}=\left(1+\frac{\sum_{i=1}^{n}\left(Q_{o b s, i}^{\lambda}-Q_{s i m, i}^{\lambda}\right)^{2}}{\sum_{i=1}^{n}\left(Q_{o b s, i}^{\lambda}-\overline{Q_{o b s}^{\lambda}}\right)^{2}}\right) *\left(1+\sum_{k=1}^{m}\left|\sum_{j=1}^{365} \frac{\left(Q_{s i m, j}-Q_{o b s, j}\right)}{\sum_{j=1}^{365} Q_{o b s, j}}\right| * \frac{1}{m}\right)
$$

where $m$ is the number of 365 day segments the simulated and observed time series are sub-divided into. For this study the $\lambda$ value used was 0.5 The scale of aggregation was chosen to be 365 to approximate annual bias. This objective function has been shown to improve the predictive performance of the GR7J model relative to other objective functions (Hughes et al., 2015).

\section{RESULTS AND DISCUSSION}

Examination of catchment data, namely the $P E, P$ and $Q$ time series allow examination of the catchment observations in reference to the mass balance limits (Figure 2). All except three catchments fall with reasonable mass balance limits. The possibility exists that these three catchments are losing water to surrounding catchments, although measurement error or catchment area error are possible also. However, unlike the study of Le Moine et al. (2007), there appears little evidence for groundwater exchange based upon mass balance observations.

The calibrated $x_{2}$ value was generally negative across the 137 study catchments. For GR4J $x_{2}$ was negative for $81 \%$ of catchments while GR7J $x_{2}$ was negative in $78 \%$ of catchments. So, in most cases, water was "lost" from simulations. If it is assumed that no groundwater exchange can be justified, then this process is substituting for evaporative processes in simulations with negative values of $x_{2}$. Given the mass balance of catchments it is also very difficult to justify positive $x_{2}$ values since $\mathrm{Q} / \mathrm{P}$ ratios rarely exceed 0.6 for the study catchments (Figure 2). Model fluxes for simulations with negative $x_{2}$ values show that groundwater exchange out of the catchment (denoted GW loss) is quite high relative to catchment evapo-transpiration (ET - Figure 3). Mean groundwater loss in simulations was 63 and $46 \mathrm{~mm} / \mathrm{yr}$ for GR7J and GR4J respectively. These are significant proportion of the water budget but are difficult to justify conceptually. It is possible that some water 
J. D. Hughes et al., Inter-basin groundwater exchange...

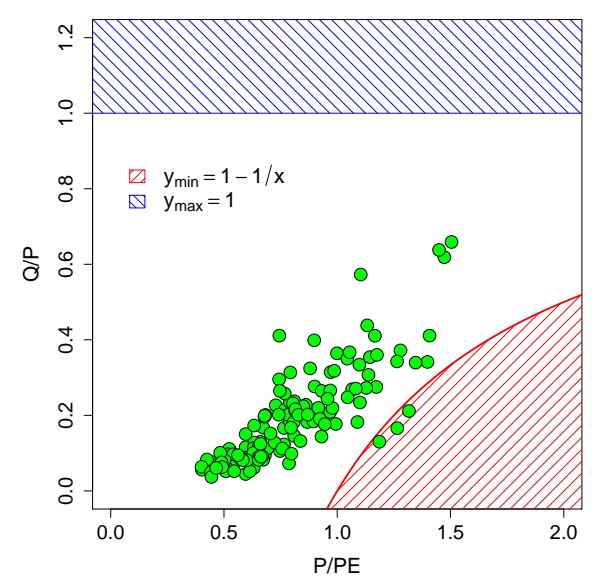

Figure 2. Non-dimensional graphical representation of study catchments described by their mean streamflow $\mathrm{Q}$, mean precipitation $\mathrm{P}$, and mean potential evapo-transpiration $\mathrm{PE}$.

is lost via recharge of deeper regional aquifers although the values obtained in this study (Figure 3), appear to be higher than recharge studies suggest (Barron et al., 2011). Examination of mean GW exchange values for simulations along with climate and parameter values showed that simulated GW exchange was higher for more humid catchments (Figure 4). There data indicate there is little correlation between the $x_{2}$ parameter and simulated GW exchange. Rather, this is related to the $x_{3}$ parameter, value that is higher for catchments with a PE/P ratio of less than 1.5.

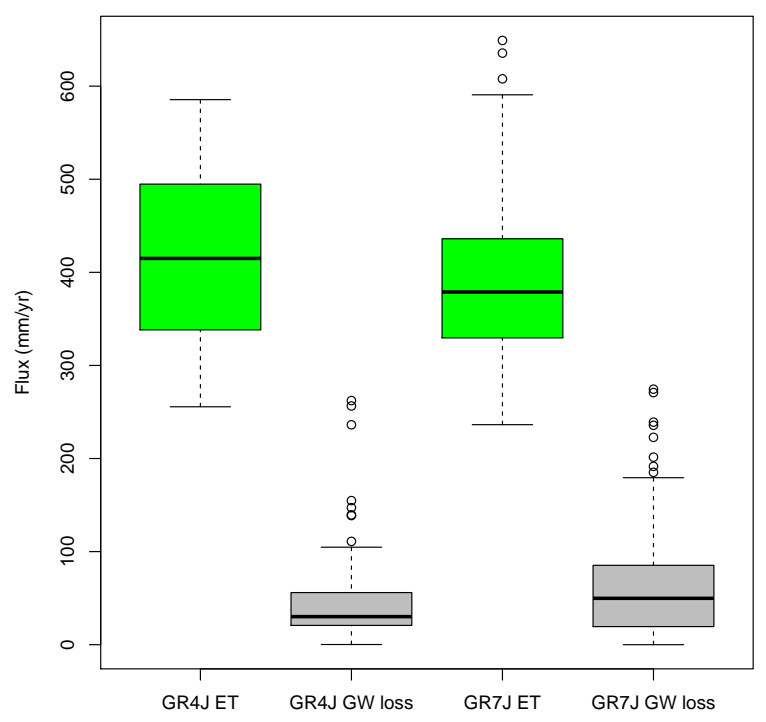

Figure 3. Simulated mean annual evapo-transpiration and groundwater exchange (loss) for GR4J and GR7J models with negative $x_{2}$ values. 
J. D. Hughes et al., Inter-basin groundwater exchange...
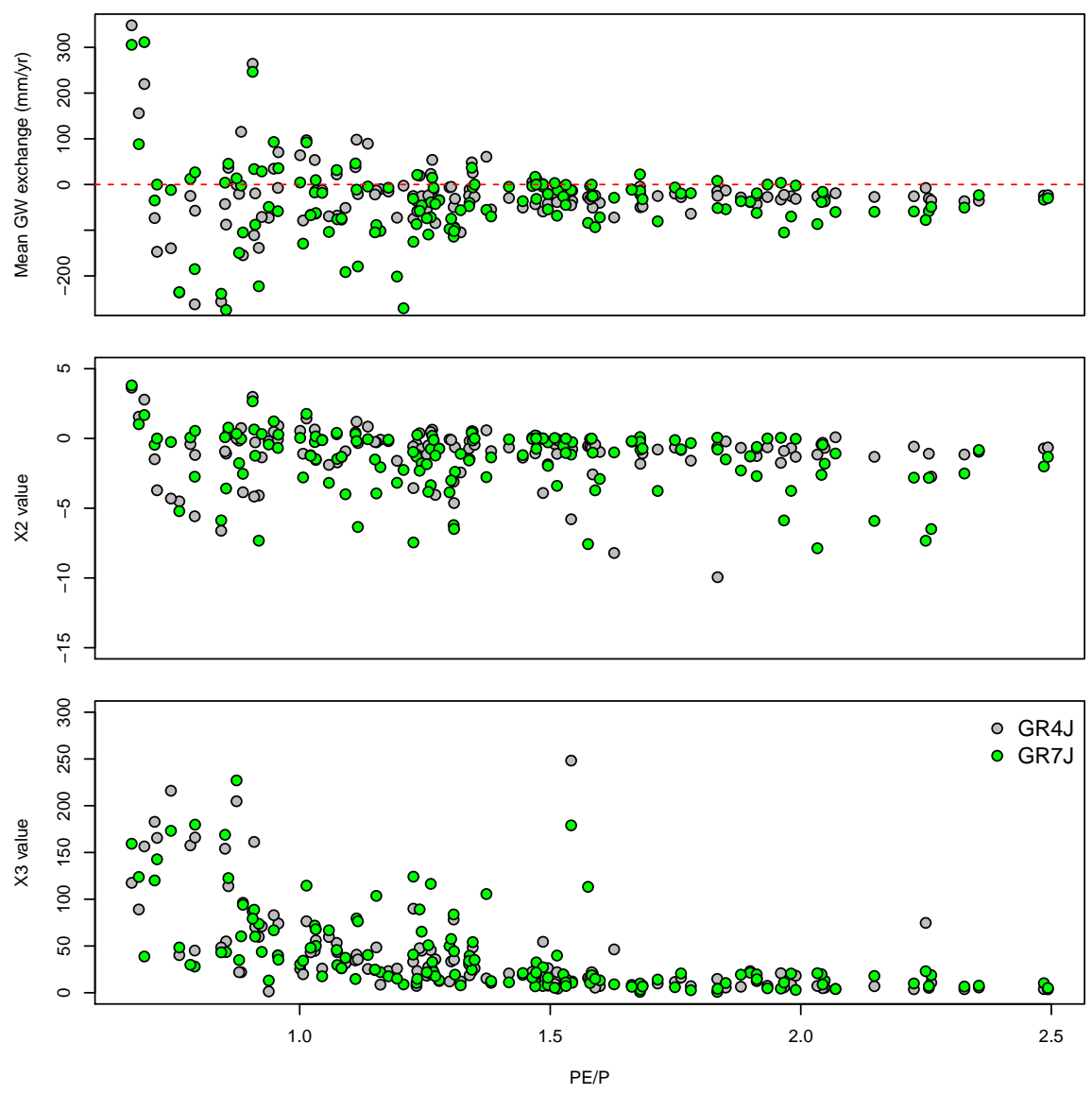

Figure 4. The relationship between simulated mean GW exchange, calibrated $x_{2}$ values, calibrated $x_{3}$ values and catchment aridity (PE/P).

To examine the effect of switching of the groundwater exchange process, a random selection of 30 catchments (from the original 137) were re-calibrated with $x_{2}$ to zero. This reduced the calibration goodness of fit in both models, and increased the mean absolute value of simulation bias in GR4J during calibration (Figure 5). Setting $x_{2}$ had little effect on calibration bias in GR7J. Conversely, evaluation NSE was improved in GR7J by setting $x_{2}$ to zero, with the opposite effect in GR4J. Similarly absolute mean bias was increased for GR4J in evaluation when $x_{2}$ was set to zero, while GR7J bias remained largely unaffected. This indicates the possibility that the GR7J model could be either reduced to six parameters or that the $x_{2}$ parameter could be set to zero where there is little possibility of inter-catchment groundwater transfer. Given the poor performance of GR4J with $x_{2}$ set to zero it could not be used in the same way.

\section{CONCLUSIONS}

Analysis of catchment climate and runoff data for 137 catchments in eastern Australia indicates that the likelihood of inter-catchment groundwater transfer appears low. The evaporation term of catchment water balance is much higher than in European catchments, making water balance errors more difficult to detect. Despite this both GR4J and GR7J calibrate to non-zero values. Around $80 \%$ of calibrated models produce negative $x_{2}$ values, resulting in significant groundwater fluxes out of the catchment. Assuming no groundwater exchange is present, setting the $x_{2}$ value to zero will degrade the predictive performance of GR4J but has no degradation of the GR7J model assuming the appropriate parameter bounds are allowed during calibration. 
J. D. Hughes et al., Inter-basin groundwater exchange...
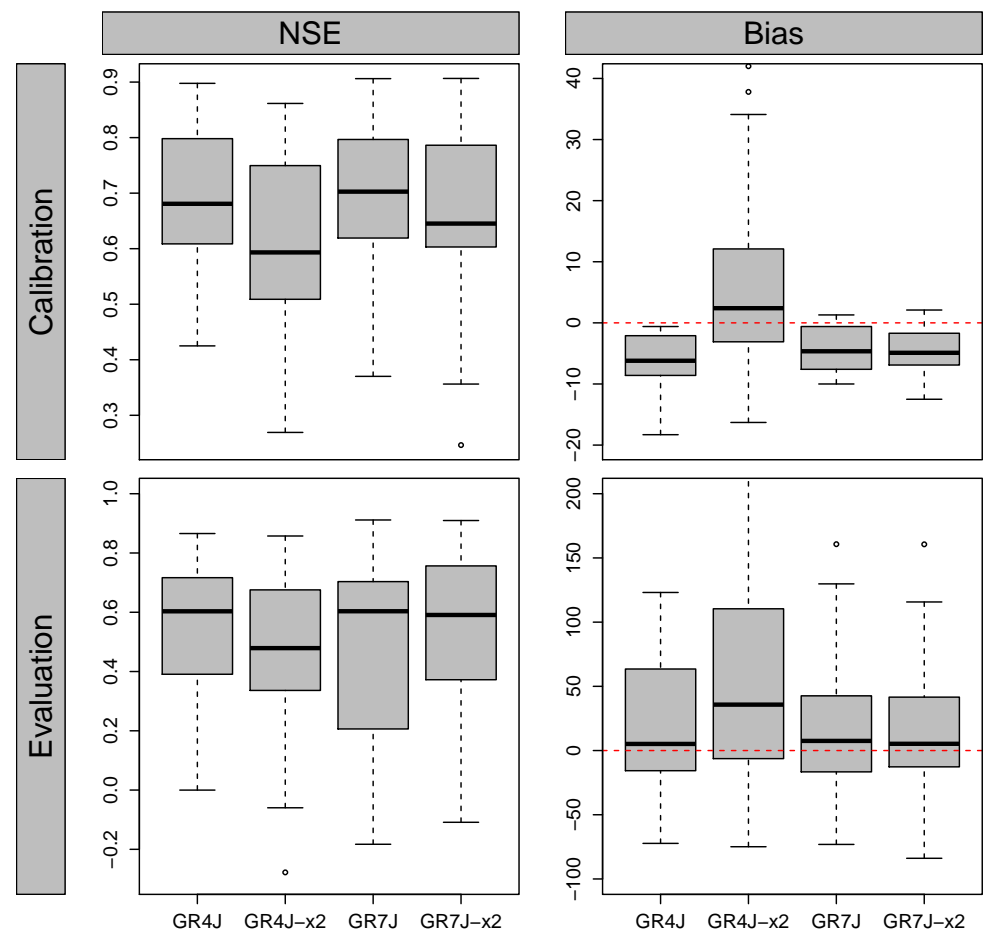

Figure 5. The effect of setting the $x_{2}$ parameter to zero on model goodness of fit during calibration and drought evaluation. Models with $x_{2}$ set to zero are denoted "-x2". Bias is expressed as a percentage.

\section{ACKNOWLEDGEMENT}

The authors would like to thank Robert Bridgart for his assistance with coding the GR7J model and JeanMichel Peraud for the production of the R package $r C l r$ that was critical to this study.

\section{REFERENCES}

Barron, O., R. Crosbie, S. Charles, W. R. J. Dawes, R. Ali, W. R. Evans, R. G. Cresswell, D. W. Pollock, G. Hodgson, D. Currie, et al. (2011). Climate change impact on groundwater resources in Australia. National Water Commission.

Hughes, J. D., N. J. Potter, L. Zhang, and R. Bridgart (2015). Conceptual model modification and runoff generation in the millennium drought of southeastern Australia. Journal of Hydrology, In review.

Le Moine, N., V. Andreassian, C. Perrin, and C. Michel (2007). How can rainfall-runoff models handle intercatchment groundwater flows? Theoretical study based on 1040 French catchments. Water Resources Research 43(W06428).

Perrin, C., C. Michael, and V. Andreassian (2003). Improvement of a parsimonious model for streamflow simulations. Journal of Hydrology 279, 275-289.

Saft, M., A. W. Western, L. Zhang, M. C. Peel, and N. J. Potter (2015). How does multiyear drought influence the rainfall-runoff relationship. Water Resources Research submitted.

Vaze, J., D. A. Post, F. H. S. Chiew, J. M. Perraud, N. R. Viney, and J. Teng (2010). Climate non-stationarity - Validity of calibrated rainfall-runoff models for use in climate change studies. Journal of Hydrology 394, 447-457. 